Published in final edited form as:

Psychol Med. 2014 July ; 44(9): 1913-1925. doi:10.1017/S0033291713002638.

\title{
A Swedish national adoption study of criminality
}

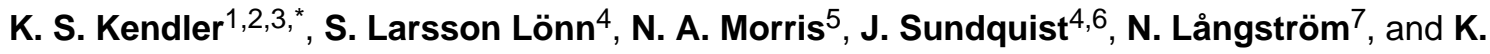 \\ Sundquist ${ }^{4,6}$ \\ ${ }^{1}$ Virginia Institute for Psychiatric and Behavioral Genetics, Virginia Commonwealth University, \\ Richmond, VA, USA \\ 2Department of Psychiatry, Virginia Commonwealth University, Richmond, VA, USA \\ ${ }^{3}$ Department of Human and Molecular Genetics, Virginia Commonwealth University, Richmond, \\ VA, USA \\ ${ }^{4}$ Center for Primary Health Care Research, Lund University, Malmö, Sweden \\ ${ }^{5}$ Department of Criminal Justice, Virginia Commonwealth University, Richmond, VA, USA \\ ${ }^{6}$ Stanford Prevention Research Center, Stanford University School of Medicine, Stanford, CA, \\ USA
}

${ }^{7}$ Department of Medical Epidemiology and Biostatistics, Karolinska Institute, Stockholm, Sweden

\section{Abstract}

Background-To clarify the role of genetic and environmental factors in criminal behavior (CB), we examined all CB and violent and non-violent subtypes (VCB and NVCB, respectively) in a Swedish national sample of adoptees and their relatives.

Method-CB was defined by a conviction in the Swedish Crime Register with standard definitions for VCB and NVCB subtypes. We examined adoptees born 1950-1991 ( $n=18070)$ and their biological $(n=79206)$ and adoptive $(n=47311)$ relatives.

Results-The risk for all CB was significantly elevated in the adopted-away offspring of biological parents of which at least one had CB [odds ratio (OR) 1.5, 95\% confidence interval (CI) 1.4-1.6] and in the biological full and half-siblings of CB adoptees (OR 1.4, 95\% CI 1.2-1.6 and OR 1.3, 95\% CI 1.2-1.3, respectively). A genetic risk index (including biological parental/sibling history of $\mathrm{CB}$ and alcohol abuse) and an environmental risk index (including adoptive parental and sibling CB and a history of adoptive parental divorce, death, and medical illness) both strongly predicted probability of $\mathrm{CB}$. These genetic and environmental risk indices acted additively on adoptee risk for CB. Moderate specificity was seen in the transmission of genetic risk for VCB and NVCB between biological parents and siblings and adoptees.

(C) Cambridge University Press 2013

*Address for correspondence: K. S. Kendler, M.D., Virginia Institute for Psychiatric and Behavioral Genetics of VCU, Box 980126, Richmond, VA 23298-0126, USA. (kendler@vcu.edu).

Declaration of Interest

None. 
Conclusions-CB is etiologically complex and influenced by a range of genetic risk factors including a specific liability to $\mathrm{CB}$ and a vulnerability to broader externalizing behaviors, and by features of the adoptive environment including parental $\mathrm{CB}$, divorce and death. Genetic risk factors for VCB and NVCB may be at least partially distinct.

\section{Keywords}

Adoption studies; crime; environment; genetics; non-violent crime; Sweden; violent crime

\section{Introduction}

Understanding the roots of human criminal and violent behavior has been a fundamental question in the social and biomedical sciences since the mid-19th century (Lilly et al. 2010). In light of the strong evidence that criminal and violent behaviors 'run in families' (Burt, 1925; Glueck \& Glueck, 1950; Farrington et al. 1975), a central subject in the large resulting research literature has been determining the magnitude of the genetic contributions to the propensity to crime. A long tradition of genetic epidemiological research has addressed this question (Lange, 1929; Rosanoff et al. 1934). For example, classic twin studies using national registers in Denmark (Christiansen, 1974) and Norway (Dalgard \& Kringlen, 1976) found heritable influences on broadly defined criminal behavior (CB) (including both violent and non-violent $\mathrm{CB}$; VCB and $\mathrm{NVCB}$, respectively), and many other investigators found genetic influences on a range of antisocial/aggressive disorders and traits (Mason \& Frick, 1994; Rhee \& Waldman, 2002; Frisell et al. 2011). However, findings on criminality from adoption studies, the most powerful design in humans to separate 'nature and nurture', have been surprisingly inconclusive. Given the potential methodological limitations of the non-experimental designs possible in human genetics, it is particularly important to attempt to validate findings using different and complementary methods.

The first adoption study of broadly defined CB identified 52 adopted-away offspring of women with criminal offences in Iowa and 52 matched adoptive controls, and reported a significant excess of criminal records and incarceration in the index versus control adoptees (Crowe, 1972). The largest study to date, performed with the Danish adoption register and examining only males (13194 adoptees), found evidence for genetic transmission of risk to property crime but not to violent crime as well as an association between all convictions and the adoptive parents' social class (Gabrielli \& Mednick, 1984; Mednick et al. 1984). The Stockholm adoption study (2000 adoptees) found that criminality alone was not transmitted from biological parents to adoptees, but did find elevated rates of criminality in adoptedaway offspring of biological parents with alcohol use disorders (AUD) alone, or with both AUD and criminality (Bohman, 1978). Very recently, self-report measures of CB were assessed in a small US sample of adoptees (about 250 subjects) and found to be significantly and positively correlated with adoptee reports about CB in their biological parents (Beaver, 2011).

We here report results of an analysis of total CB and the two subtypes of CB, i.e. VCB and NVCB, in a Swedish nationwide adoption cohort of 18070 adoptees and their 79206 biological and 47311 adoptive relatives. We address the following questions: 
1. Do genetic factors make an impact on risk for total $\mathrm{CB}, \mathrm{VCB}$ and $\mathrm{NVCB}$ ?

2. If so, are there other features such as a history of AUD or drug abuse (DA) in the biological parents and siblings that predispose to CB in the adoptees? As suggested in a previous Swedish adoption study (Bohman, 1978), are the genetic effects on CB entirely explained by the genetic risk for AUD?

3. Is there evidence for specificity of genetic risk for VCB versus NVCB?

4. Do environmental features of the adoptive home influence risk for $\mathrm{CB}$ and are there differences in the risks for VCB versus NVCB?

5. Do the genetic and environmental risk factors for CB, VCB and NVCB add together in their effects or do they interact?

\section{Method}

We linked nationwide Swedish registers via the unique 10-digit identification number assigned at birth or immigration to all Swedish residents. The identification number was replaced by a serial number to ensure anonymity.

The following sources were used to create the data-sets analysed here: The Crime Register containing all convictions in the lower court from 1973 to 2011; the Hospital Discharge Register including all hospitalizations in Sweden from 1964 to 2009; the Outpatient Care Register containing information from all out-patient physician care in Sweden from 2001 to 2009 (excluding primary healthcare); the Prescribed Drug Register covering all prescriptions picked up by patients from 1 June 2005 to 2009; the Primary Health Care Register including out-patient care diagnosis with partial coverage of the Swedish population with data from 2001 to 2007; the Cause of Death Register containing causes of death from 1961 to 2007; the Suspicion Register containing information of individuals suspected of crime until 2012; the Swedish Censuses from 1960 1970, 1980 and 1990; the Total Population Register including annual data on individual education and marital status from 1990 to 2009. Adoptive and biological relations were identified through the Multi-Generation Register providing information of family relations of individuals born in Sweden after 1932 and ever registered as living in Sweden after 1960. There is similar information for immigrants who became citizens of Sweden before the age of 18 years together with one or both parents. The study population consisted of all individuals that could be identified in the Multi-Generation Register. We constrained the population to individuals born at the latest 1991 to allow for follow-up time at risk, as the age for criminal responsibility in Sweden is 15 years, recorded from 1950 on.

\section{Measures}

Various types of $\mathrm{CB}$ were identified in the Crime register and represented as dichotomous outcomes. VCB was defined as having had one or more of the following criminal convictions (numbers within parentheses are law chapters and paragraphs): (aggravated) assault (3:5, 3:6); illegal threat (4:5); threats and violence against an officer (17:1, 17:2); intimidation (4:7); (gross) violation of a person's/woman's integrity (4:4a); kidnapping (4:1); illegal confinement or restraint (4:2); (aggravated) robbery $(8: 5,8: 6)$; illegal coercion 
(4:4); (aggravated) arson (13:1, 13:2); murder, manslaughter or filicide (3:1, 3:2, 3:3); and sexual crimes (excluding prostitution and the buying of sexual services but including child pornography) (6:1-6:10, 6:12, 16:10A). The following criminal convictions were considered non-violent: theft of a vehicle (8:1-2, 8:4, 8:7-8); theft (including burglary) (8:1-2, 8:4); vandalism (12:1-4); vandalism causing danger to the public, sabotage, hijacking [13:3-10 $(5 \mathrm{a}-\mathrm{b})$ ]; unlawful entering of a person's home, trespassing (4:6); fraud (9:1-10); embezzlement [10:1-8 (5a-e)]; dishonesty/ crime towards a creditor (includes forged bookkeeping in companies) (11:1-5); and forgery (14:1-10). In our adoptee analyses, we utilized a hierarchy such that an individual with VCB may or may not have also had a conviction for a non-violent crime, while an individual with NVCB was defined as never having had a conviction for a violent crime. CB was defined as any of these two types. However, for ease of comparability across analyses, in the biological and adoptive relatives, VCB and NVCB were defined non-hierarchically so that an individual with NVCB may or may not have also had a conviction for a violent crime. Finally, in our analysis of NVCB in adoptees, adoptees with VCB were excluded from the analysis since considering them 'unaffected' was not sensible.

AUD was identified in the Hospital Discharge Register from International Classification of Diseases (ICD) codes [ICD-8 codes for alcoholism (303) and alcoholic psychosis (291); ICD-9 codes for alcohol dependence syndrome (303) and alcoholic psychosis (291); and ICD-10 codes for mental and behavioral disorder due to the use of alcohol (F10)]. DA was identified in the Swedish Medical Registries by ICD codes [ICD-8: drug dependence (304); ICD-9: drug psychoses (292) and drug dependence (304); ICD-10: mental and behavioral disorders due to psychoactive substance use (F10-F19), except those due to alcohol (F10) or tobacco (F17)]; in the Suspicion Register by codes 3070, 5010, 5011 and 5012, which reflect crimes related to DA; and in the Crime Register by references to laws covering narcotics (law 1968:64, paragraph 1, point 6), and drug-related driving offences (law 1951:649, paragraph 4, subsection 2 and paragraph 4A, subsection 2). DA was also identified in individuals (excluding those suffering from cancer) by Anatomical Therapeutic Chemical (ATC) codes in the Prescribed Drug Register who had retrieved (on average) more than four defined daily doses per day for 12 months from either hypnotics, sedatives (N05C and N05BA) or opioids (N02A).

Psychiatric illness was defined from the main diagnosis in the Swedish Medical Registries by ICD codes for the following diagnoses: ICD-8 codes: 295-302 and 305-307; ICD-9 codes 295-298, 300-302, 306-309, 311, and 312; ICD-10 codes F20-F25, F28-F34, F38F45, F48, F50-F54, F59-F69 and F99.

\section{Sample}

The study population consisted of individuals born 1950-1991 that had been adopted with information available on both adoptive and at least one biological parent. Individuals adopted by biological relatives (sibling, sibling of parents or grandparents) or by a stepparent living with a biological parent were excluded. Age at formal adoption was not available in national records until 1991. We therefore estimated age at first cohabitation with adoptive parents (AFCAP) from census data, including individual addresses, available every 
5 years (e.g. 1950, 1955, 1960, etc.). For an adoptee born in 1961 and living with adoptive parents in the 1965 census, AFCAP was calculated as 4 years, although it could have been from 0 to 4 years. Thus AFCAP represents an upper limit of the true age at adoption.

\section{Genetic risk variables}

We utilized the following variables for biological parents and/or biological siblings, measured during the entire life course (as covered in national registers): $\mathrm{CB}, \mathrm{VCB}, \mathrm{NVCB}$, AUD, DA, and any psychiatric illness. Among parents, at least one parent had to be defined according to the above criteria. For siblings, we created a weighted score from the number of full and half-siblings, with the former weighted twice as much as the latter, to reflect their genetic relatedness to the adoptee. If there were no siblings, this variable was set to zero.

In parents, we considered educational attainment as a proxy for socio-economic status. To control for cohort effects, we defined low and high educational attainment as below or above the 75th percentile of the distribution of years of education in the decade of birth for the entire Swedish population. We also considered divorces among biological mothers (if no biological mother or missing marital status for mother, the father's status was used) during the entire life course. Finally, we considered the mother's age at birth of the adoptee. If mother's age was missing, the biological father's age was used.

\section{Environmental risk variables}

We utilized the following variables in adoptive parents from AFCAP until the adoptee was 20 years old: CB, VCB, NVCB, AUD, DA, psychiatric illness, and divorce. We added hospitalization for any medical problem and death (from the mortality register). We also considered education as a proxy for socioeconomic status, using the highest education of the adoptive mother or father. We also considered age of the adoptive mother at the time of adoption.

The following variables were considered among adoptive siblings and were measured during their life course as covered in national registers (for definition of variables, see above): $\mathrm{CB}$, VCB, NVCB, DA, AUD, psychiatric illness and other medical problems. We created a score weighted linearly according to the number of siblings.

\section{Environmental and genetic risk scores}

We performed a logistic regression on the entire sample of 18070 adoptees and used all genetic and environmental variables to model increased genetic and environmental risk for $\mathrm{CB}, \mathrm{VCB}$ and NVCB. For example, we examined all genetic risk factors that were significantly associated with the outcome $(p<0.10)$ in univariate analyses and included these in multivariate logistic regressions predicting risk for $\mathrm{CB}, \mathrm{VCB}$ and $\mathrm{NVCB}$. We then obtained predicted probabilities from the multivariate analysis for each adoptee and categorized them into groups by deciles (representing our genetic risk scores) and used the decile assignment as a continuous variable reflecting genetic risk. The same procedure was performed for all variables related to adoptive parents/siblings to create an environmental risk score. 


\section{Statistical analysis}

We performed a number of statistical analyses to address our hypotheses. First, we used genetic and environmental risk scores to predict risk for CB, as well as VCB and NVCB. Because our outcomes of $\mathrm{CB}$ were dichotomous, we used logistic regression and modeled criminal behavioral outcomes as a function of the genetic risk score, the environmental risk score, sex of the adoptee, AFCAP and birth year. Second, we modeled each type of criminal behavioral outcome as a function of specific genetic and environmental risk factors, and examined if there was evidence of specificity of genetic and environmental risk factors for VCB and NVCB. In this part of the analysis, we focus on both the specificity of genetic or environmental risk factors, as well as the extent to which non-CB-related genetic and environmental risk factors (e.g. AUD or DA) make an impact on adoptee risk of CBs. Finally, as we wanted to investigate the interaction between genetic and environmental risk scores on an additive scale, we also utilized the identity link corresponding to a linear model. We estimated generalized linear models with PROC GENMOD in SAS (SAS Institute, 2007) for all regression analyses. This analysis was repeated for all three outcomes except that when modeling NVCB, adoptees with VCB but not NVCB were censored.

\section{Results}

\section{General description}

The general characteristics of our study sample are outlined in Table 1. In the 18070 adoptees (52.2\% males), whose average age in 2011 was 49.3 (S.D.=8.3) years, the prevalence rates of $\mathrm{CB}, \mathrm{VCB}$ and $\mathrm{NVCB}$ were $21.2,8.3$ and 13.2\%, respectively. Compared with adoptive parents, biological parents had higher rates of crime, AUD, DA and psychiatric illness. Similar differences were seen between the adoptive and biological full and half-siblings of the adoptees. The mean AFCAP was 4.4 (S.D.=2.7) years.

\section{All criminal behavior}

The risk for $\mathrm{CB}$ in the adopted-away offspring of biological parents of which at least one had CB was substantially elevated [odds ratio (OR) 1.5, 95\% confidence interval (CI) $1.3-$ $1.6, \chi^{2}=90.1$, degrees of freedom $\left.(\mathrm{df})=1, p<0.0001\right]$. The risk for $\mathrm{CB}$ was modestly but significantly higher in the adopted-away offspring of biological mothers $(27.9 \%, 95 \% \mathrm{CI}$ 26.2-29.7\%) than biological fathers with CB $(25.2 \%, 95 \%$ CI $23.6-26.8 \%, p<0.05)$ and was significantly increased in the biological full and half-siblings of adoptees with CB (OR 1.4, $95 \%$ CI 1.2-1.6, $\chi^{2}=28.5, \mathrm{df}=1, p<0.0001$; and OR 1.3, 95\% CI 1.2-1.3, $\chi^{2}=94.7, \mathrm{df}=1$, $p<0.0001$, respectively).

We created indices of environmental and genetic risk for CB from available characteristics of, respectively, the adoptees' biological parents and siblings, and their adoptive parents and siblings. As seen in Table 2, in univariate analyses, environmental risk for CB was predicted by a wide range of characteristics of the adoptive family including a parental history of $\mathrm{CB}$, AUD and DA, divorce, young maternal age, and premature death or medical hospitalization as well as a history of CB, AUD, DA and psychiatric or medical illness in the adoptive siblings. Genetic risk for CB was predicted, in univariate analyses, by a very similar set of 
variables including biological parental or sibling histories of VCB, DA, and psychiatric illness and parental divorce, young maternal age at childbirth and low education.

In multivariate analyses, the strongest environmental risk predictors for $\mathrm{CB}$ were adoptive parental AUD, divorce, DA and CB, and adoptive sibling CB and DA. The strongest genetic risk predictors for $\mathrm{CB}$ were $\mathrm{CB}$ and $\mathrm{AUD}$ in biological siblings and parents.

Although not included in Table 2, in univariate analyses, $\mathrm{CB}$ was slightly more strongly predicted by a history of NVCB than VCB in biological parents (OR 1.50, 95\% CI 1.381.62 and OR 1.36, 95\% CI 1.22-1.52, respectively, both $p<0.0001)$ with the reverse pattern seen in biological siblings (OR 1.71, 95\% CI 1.48-1.97 and OR 2.04, 95\% CI 1.70-2.47, respectively, both $p<0.0001)$.

We then created genetic and environmental risk indices from our multivariate analyses (Table 2) that we divided into deciles. Both risk scores were significantly predictive of CB: OR (per decile) $=1.10$ and 1.06, respectively (Table 3). (Thus, the ORs for CB between individuals at the lowest and highest deciles of genetic and environmental risk equaled $1.10^{9}$ or 2.36 and $1.06^{9}$ or 1.69 , respectively.) The correlation between risk scores, which may reflect assortative placement in the adoption process, was small (+0.12) but significant $(p<0.001)$. Examining predictors individually, $\mathrm{CB}$ in the adoptee was significantly predicted by male sex and lower AFCAP, but not by birth year. In a multivariate analysis, however (Table 3), in addition to the genetic and environmental risk indices, male sex, earlier birth year and AFCAP were all significant predictors of CB.

Finally, we analysed these significant predictor variables on the scale of raw probabilities without and with an interaction between the genetic and environmental risk scores (Table 4). The interaction term was very small and non-significant indicating that, in this sample, genetic and environmental risks made an impact additively on the probability that an adoptee was registered with CB.

\section{Violent criminal behavior}

The risk for VCB in the adopted-away offspring of biological parents of which at least one had been registered for VCB was substantially elevated (OR 1.5, 95\% CI 1.3-1.7, $\chi^{2}=28.8$, $\mathrm{df}=1, p<0.00001)$ and did not differ in the adopted-away offspring of biological mothers $(12.8 \%, 95 \%$ CI $8.5-13.8 \%)$ and biological fathers with VCB (11.1\%, 95\% CI. 4.0-12.7\%). The risk for VCB was increased similarly in biological full and half-siblings of adoptees with VCB (OR 1.5, 95\% CI 1.2-1.9, $\chi^{2}=11.9, \mathrm{df}=1, p=0.0006$; and OR 1.5, 95\% CI 1.3-1.6, $\chi^{2}=62.8, \mathrm{df}=1, p<0.0001$ respectively).

As for all CB, we next created indices of genetic and environmental risk for VCB from available characteristics of the adoptees' biological parents and siblings, and adoptive parents and siblings. In univariate analyses, environmental risk for VCB was significantly predicted by adoptive parental death, divorce, hospitalization, young age, low education, and adoptive sibling DA (Table 5). In multivariate analyses, the strongest predictors were adoptive sibling DA, and parental death, divorce and low education. Genetic risk was significantly predicted, in univariate analyses, by a much broader array of variables (Table 
5) with the strongest predictors, in the multivariate model, from biological sibling VCB and AUD, and biological parental divorce, AUD, low education and VCB. Of note, in the multivariate analyses, genetic risk for VCB was more strongly predicted by VCB than by NVCB in both biological parents and siblings.

As with all CB, risk for VCB was predicted by genetic and environmental risk scores: OR (per decile) $=1.11$ and 1.06, respectively (Table 3). (Thus, the ORs for VCB between individuals at the lowest and highest deciles of genetic and environmental risk equaled $1.11^{9}$ or 2.63 and $1.06^{9}$ or 1.69 , respectively). The correlation between risk scores was small $(+0.11)$ but significant $(p<0.001)$. Examining predictors individually, VCB in the adoptee was also significantly predicted by male sex, but not by birth year or AFCAP. In a multivariate analysis, however (Table 3), in addition to the genetic and environmental risk indices, both sex and birth year were significant predictors of VCB.

As with all $\mathrm{CB}$, we examined the genetic and environmental predictor variables on the scale of raw probabilities without and with an interaction between the genetic and environmental risk scores (Table 4). The interaction term was zero to three decimal places, indicating that genetic and environmental risks made an impact additively on the probability that an adoptee was registered for VCB.

\section{NVCB}

The risk for NVCB in the adopted-away offspring of biological parents of which at least one had NVCB was elevated (OR 1.5, 95\% CI 1.4-1.6, $\chi^{2}=66.8, \mathrm{df}=1, p<0.0001$ ). The risk for NVCB was significantly higher in the adopted-away offspring of biological mothers (19.8\%, 95\% 18.1-21.5\%) versus biological fathers with NVCB (17.3\%, 95\% 15.7-18.9\%, $p=0.043)$. The risk for NVCB was significantly increased in both biological full and halfsiblings of adoptees with NVCB (OR 1.5, 95\% CI 1.3-1.7, $\chi^{2}=32.8, \mathrm{df}=1, p<0.0001$; and OR $1.3,95 \%$ CI $1.2-1.3, \chi^{2}=79.4, \mathrm{df}=1, p<0.0001$ respectively).

As seen in Table 6, in univariate analyses, environmental risk for NVCB was significantly predicted by a broad array of features of the adoptive family, with the strongest predictors in multivariate analyses being adoptive parent DA, VCB, AUD and divorce, and adoptive sibling VCB. Genetic risk was also significantly predicted, in univariate analyses, by a wide array of variables, with the strongest predictors, in the multivariate model, being biological parental and sibling NVCB, and biological sibling VCB and AUD. In the multivariate analyses, genetic risk for NVCB was more strongly predicted by NVCB than by VCB in biological parents and a similar but more modest difference was seen in biological siblings.

The risk for NVCB was predicted by genetic and environmental risk scores: OR (per decile) $=1.09$ and 1.06, respectively (Table 3). (Thus, the ORs for VCB between individuals at the lowest and highest deciles of genetic and environmental risk equaled $1.09^{9}$ or 2.17 and $1.06^{9}$ or 1.69 , respectively.) The correlation between risk scores was small (+0.12) but significant $(p<0.001)$. Examining predictors individually, NVCB in the adoptee was also significantly predicted by male sex and AFCAP, but not by birth year. In a multivariate analysis, however, in addition to the genetic and environmental risk indices, sex, birth year and AFCAP were all significant predictors of VCB. 
Finally, we examined the genetic and environmental predictors of NVCB on the scale of raw probabilities without and with an interaction between the genetic and environmental risk scores (Table 4). As with all CB and VCB, we found no evidence of an interaction.

\section{Discussion}

We sought, through an examination of CB in a Swedish national sample of adoptees and their biological and adoptive relatives, to clarify the sources of individual differences in risk for criminal and violent behavior. We had five specific aims in this paper, which we now review in turn.

First, consistent with prior twin studies of CB and a range of other related externalizing traits (Christiansen, 1974; Dalgard \& Kringlen, 1976; Rhee \& Waldman, 2002), we found robust evidence for the genetic transmission of all CB as well as VCB and NVCB subtypes. Our evidence came from both traditional adoption designs: an increased risk for all $\mathrm{CB}$, VCB and NVCB in (i) adopted-away children of affected parents and in (ii) biological siblings of affected adoptees. Notably, these analyses suggested that the strength of the genetic effect on VCB and NVCB was similar. Our results were not congruent with the prior largest adoption study of criminality that found, in a Danish adoption cohort, genetic transmission of non-violent property but not of violent offenses (Mednick et al. 1984).

Second, CB in the adoptee was predicted by other features in the biological parents and siblings beyond their own history of $\mathrm{CB}$. A history of DA in biological parents and siblings predicted all forms of $\mathrm{CB}$ in univariate analyses but were no longer significant in the multivariate results. For all CB and VCB, AUD in both biological parents and siblings were strong predictors in univariate analyses that remained significant in multivariate results. Interestingly, AUD in biological relatives was a much weaker predictor of NVCB. This is consistent with prior studies showing that children of alcoholic parents are at a high risk of experiencing externalizing behaviors and problem behaviors marked by increased aggression and impulsivity (Scher, 1991; Eiden et al. 2007).

Psychiatric illness in biological relatives was a relatively weak predictor for CB in adoptees that typically was no longer significant in multivariate analyses. Interestingly, both young maternal age at birth and a history of maternal divorce were robust predictors of risk for all CB as well as VCB and NVCB. Inconsistent with results from the Stockholm adoption sample (Bohman, 1978), our multivariate analyses showed that the genetic transmission of risk for all CB, as well as VCB and NVCB subtypes, between biological parents and adopted children, and adoptees and their biological siblings, was not explained by the genetic transmission of AUD.

Third, we found, in multivariate analyses, consistent evidence for specificity in the genetic transmission of risk for VCB versus NVCB. VCB was more strongly predicted in adoptedaway children by VCB than by NVCB in biological parents, and VCB in the adoptee more strongly predicted risk for VCB than NVCB in biological siblings (not reared with the adoptee). More dramatically, controlling for NVCB, a history of VCB in biological parents was unrelated to risk for NVCB in adopted-away offspring, with a similar but less striking 
pattern of results seen in biological siblings. These findings are in line with the research examining biosocial causes of crime which indicates that there are a number of genetic and congenital factors associated with the transmission of violence in particular (Raine, 1993; Raine et al. 1994; Tibbetts \& Piquero, 1999), as well as studies that indicate temperamental predictors associated primarily with violent convictions (Sigvardsson et al. 1987; Henry et al. 1996). This may also lend support for developmental models that propose multiple pathways to antisocial behavior and crime (Loeber et al. 1993; Moffitt, 1993; Nagin \& Tremblay, 1999; Burt, 2012). For example, Loeber et al. (1993) propose a multiple pathway model in which distinct early childhood risk factors lead to two types of offending characterized by covert and overt criminal acts. The covert pathway predisposes to property damage, and moderate to serious forms of crime, whereas the overt pathway is typified by aggression, fighting and violence.

Fourth, consistent with a large prior literature (Mednick et al. 1984; Goetting, 1994; Fonagy et al. 1998; Hawkins et al. 1998), we found that disruption of the parent-child relationship through divorce, parental death or parental illness predisposed to CB. However, contrary to nearly all prior studies, our subjects were not genetically related to their adoptive family so that the causal effects of these environmental factors on aggressive as well as general $\mathrm{CB}$ are not confounded by genetic effects. For all CB and NVCB, but not VCB, we found evidence consistent with direct parent-offspring environmental transmission of $\mathrm{CB}$, in that adoptive parental $\mathrm{CB}$ predicted $\mathrm{CB}$ in the offspring. For all $\mathrm{CB}$, we also found evidence for shared environmental effects, as $\mathrm{CB}$ in the adoptee was significantly predictive of $\mathrm{CB}$ in the adoptive siblings. A few other results of environmental factors were of potential interest; for example, low adoptive parental education and adoptive parental death both significantly predicted VCB but not NVCB.

Fifth, we sought to determine how joint effects of genetic and environmental risk factors together affected risk for CB. Consistent with prior findings in the Danish adoption cohort (Gabrielli \& Mednick, 1984), we found no evidence for an interaction in the prediction of all CB or more specifically on VCB or NVCB forms. Hence, the impact of an adverse rearing environment on risk for $\mathrm{CB}$ was predicted to be the same in those with low versus high genetic vulnerability.

We recently completed an adoption study of DA in this same cohort using similar methods (Kendler et al. 2012). Three noteworthy differences emerged. First, genetic effects were stronger for DA with the OR of transmission from biological parent to child equaling 2.1 for DA versus about 1.5 for CB. Second, as assessed by our multivariate analyses, the impact of family environmental factors was somewhat weaker for $\mathrm{VCB}$ (OR 1.06 per decile) than for DA (OR 1.10). Third, we found gene-environment interaction for DA but not for CB. Given that $\mathrm{CB}$ is considerably more common in the Swedish population than DA, this suggests that our inability to detect gene-environment interactions for CB is probably not a result of inadequate power.

We are not the first to examine CB in the Swedish adoption data. As part of a survey of resemblance for VCB in a wide variety of relative pairs, Frisell et al. (2011) recently examined adoptive and biological relatives. Hjalmarsson \& Lindquist (2013) also recently 
studied CB in the Swedish adoption registry from an economics perspective. However, neither study excluded adoptees adopted by biological relatives, controlled for AFCAP or examined the broad array of genetic and environmental factors explored in this study. Both studies included older adoptees (born from 1932 or 1943, respectively, versus 1950 in the present study) which could bias the sample toward those with late-onset criminal convictions (because the Crime register starts in 1973). Frisell et al. (2011) did not examine NVCB, and Hjalmarsson \& Lindquist (2013) excluded females. Neither study examined the relationship between genetic and environmental risks for VCB and NVCB. However, as would be expected, our results, where similar questions were asked, are reassuringly congruent with those reported by these two prior studies (Frisell et al. 2011; Hjalmarsson \& Lindquist, 2013).

As predicted by genetic theory for additive genetic effects, the ORs for associations between $\mathrm{CB}$ in the adoptee, and the biological parents and full siblings were similar for all the definitions of $\mathrm{CB}$ that we examined. For all $\mathrm{CB}$ and NVCB, consistent with genetic expectations, the association between $\mathrm{CB}$ in adoptees was stronger for $\mathrm{CB}$ in the biological full than among biological half-siblings. Surprisingly, for VCB, these associations were similar. We have no ready explanation, other than sampling error, for this anomalous finding.

\section{Limitations}

These results should be interpreted in the context of four potential methodological limitations. First, the Swedish Crime Register contains only data on criminal convictions. As elsewhere, in Sweden, a majority of most crimes are not officially reported or do not result in a conviction. In the 2008 National Swedish Crime Victim Survey, the proportion of crimes reported to the police ranged from $14 \%$ for sexual offences to $55 \%$ for serious assaults (Swedish National Council for Crime Prevention, 2008). Bias might arise if the probability that a committed crime is reported, or that a reported crime leads to a conviction, differs across social strata or between biological and adoptive families. However, previous studies have not found this to be the case.

Second, non-random placement of adoptees can bias adoption studies. Prior studies of Swedish adoptions note, however, modest selective placement (Bohman, 1970; Bjorklund et al. 2006). Our indices of genetic and environmental risk for CB were also correlated $(+0.11$ to +0.12 ), but at a magnitude too small to substantially influence findings.

Third, the nature of adoption changed somewhat over the period of our study (1950-1991) with the wider availability of birth control and abortion, and the reduced stigma of single motherhood (Bohman, 1970).

Fourth, bias can potentially arise in adoption studies from contact between the adoptee and biological parents prior to adoption. From census records available every 5 years, we know that at least 70.5\% of adoptees were living with their adoptive parents by the age of 5 years. However, during our study period, adoptees in Sweden were typically removed from their biological mother shortly after birth and initially placed in special nurseries (Bohman, 1970; Bjorklund et al. 2006). Over this time period, in Stockholm county, $83 \%$ of adopted children 
were placed in their adoptive home by age 1 year (Hjalmarsson \& Lindquist, 2013).

Therefore, prior to adoptive placement, which tended to be early, our adoptees were more likely to be in the special nursery or foster homes than with their biological parents. We assessed this bias by examining the association between risk for CB in the adoptee and AFCAP. If sustained contact with biological parents occurred and increased risk for adoptee $\mathrm{CB}$, then AFCAP should be positively associated with CB. Instead, the association was consistently slightly negative.

\section{Conclusions}

In the largest and most complete adoption study of CB to date, we found clear evidence for the etiological complexity of $\mathrm{CB}$. The risk for $\mathrm{CB}$ was influenced by a range of genetic risk factors including a specific liability to $\mathrm{CB}$, a broad vulnerability to AUD and young age of the biological mother as well as by features of the adoptive home environment including low parental educational attainment and parental divorce and death. We found evidence for environmental transmission of CB to adoptees both from adoptive parents and adoptive siblings. There appeared to be at least moderate specificity in the genetic transmission of violent versus non-violent forms of crime. We found no evidence for gene-environmental interaction in that the environmental risk factors for $\mathrm{CB}$ had a similar impact on liability to $\mathrm{CB}$ in those at low and high genetic risk.

\section{Acknowledgments}

This project was supported by the Ellison Medical Foundation (to K.S.K., no. NR-SS-0102-12), National Institutes of Health grant no. DA030005 (to J.S.), the Swedish Research Council, the Swedish Council for Information on Alcohol and Other Drugs (CAN) as well as ALF (Government Grant for Clinical Research) funding from Region Skåne (awarded to J.S. and K.S.).

\section{References}

Beaver KM. Genetic influences on being processed through the criminal justice system: results from a sample of adoptees. Biological Psychiatry. 2011; 69:282-287. [PubMed: 21071016]

Bjorklund A, Lindahl M, Plug E. The origins of intergenerational associations: lessons from Swedish adoption data. Quarterly Journal of Economics. 2006; 121:999-1028.

Bohman, M. Adopted Children and Their Families: A Follow-up Study of Adopted Children, Their Background, Environment and Adjustment. Proprius; Stockholm: 1970.

Bohman M. Some genetic aspects of alcoholism and criminality. A population of adoptees. Archives of General Psychiatry. 1978; 35:269-276. [PubMed: 727885]

Burt SA. How do we optimally conceptualize the heterogeneity within antisocial behavior? An argument for aggressive versus non-aggressive behavioral dimensions. Clinical Psychology Review. 2012; 32:263-279. [PubMed: 22459789]

Burt, SC. The Young Delinquent. University of London Press; London: 1925.

Christiansen, KO. Seriousness of criminality and concordance among Danish twins. In: Hood, R., editor. Crime, Criminology and Public Policy. The Free Press; New York: 1974. p. 63-77.

Crowe RR. The adopted offspring of women criminal offenders. A study of their arrest records. Archives of General Psychiatry. 1972; 27:600-603. [PubMed: 5080288]

Dalgard O, Kringlen E. A Norwegian twin study of criminality. British Journal of Criminality. 1976; 16:213-232.

Eiden RD, Edwards EP, Leonard KE. A conceptual model from the development of externalizing behavior problems among kindergarten children of alcoholic families: role of parenting and children's self-regulation. Developmental Psychology. 2007; 43:1187-1201. [PubMed: 17723044] 
Farrington DP, Gundry G, West DJ. The familial transmission of criminality. Medicine Science Law. $1975 ; 15: 177-186$.

Fonagy, P.; Target, M.; Steele, M.; Steele, H. The development of violence and crime as it relates to security of attachment. In: Osofsky, JD., editor. Children in a Violent Society. Guilford Press; New York: 1998. p. 150-177.

Frisell T, Lichtenstein P, Långström N. Violent crime runs in families: a total population study of 12.5 million individuals. Psychological Medicine. 2011; 41:97-105. [PubMed: 20334717]

Gabrielli WF Jr, Mednick SA. Urban environment, genetics, and crime. Criminology. 1984; 22:645652.

Glueck, S.; Glueck, ET. Unraveling Juvenile Delinquency. 1. Harvard University Press; Cambridge, MA: 1950.

Goetting A. The parenting-crime connection. Journal of Primary Prevention. 1994; 14:169. [PubMed: 24258818]

Hawkins, JD.; Herrenkohl, T.; Farrington, DP.; Brewer, D.; Catalano, RF.; Harachi, TW. A review of predictors of youth violence. In: Loeber, R.; Farrington, DP., editors. Serious and Violent Juvenile Offenders: Risk Factors and Successful Interventions. Sage Publications, Inc; London: 1998. p. 106-146.

Henry B, Caspi A, Moffitt TE, Silva PA. Temperamental and familial predictors of violent and nonviolent criminal convictions: age 3 to age 18. Developmental Psychology. 1996; 32:614-623.

Hjalmarsson R, Lindquist MJ. The origins of intergenerational associations in crime: lessons from Swedish adoption data. Labour Economics. 2013; 20:68-81.

Kendler KS, Sundquist K, Ohlsson H, Palmer K, Maes H, Winkleby MA, Sundquist J. Genetic and familial-environmental influences on risk for drug abuse: a national Swedish adoption study. Archives of General Psychiatry. 2012; 69:690-697. [PubMed: 22393206]

Lange, J. Verbrechen als Schicksal: Studien an Kriminellen Zwillingen [Crime as Destiny: A Study on Criminal Twins]. Thieme; Leipzig: 1929.

Lilly, JR.; Cullen, FT.; Ball, RA. Criminological Theory: Context and Consequences. 5. Sage Publications, Inc; London: 2010.

Loeber R, Wung P, Keenan K, Giroux B, Stouthamer-Loeber M, Van Kammen WB, Maughan B. Developmental pathways in disruptive child behavior. Development and Psychopathology. 1993; 5:1103-1133.

Mason D, Frick P. The heritability of antisocial behavior: a meta-analysis of twin and adoption studies. Journal of Psychopathology and Behavioral Assessment. 1994; 16:301-323.

Mednick SA, Gabrielli WF Jr, Hutchings B. Genetic influences in criminal convictions: evidence from an adoption cohort. Science. 1984; 224:891-894. [PubMed: 6719119]

Moffitt TE. Adolescence-limited and life-course-persistent antisocial behavior: a developmental taxonomy. Psychology Review. 1993; 100:674-701.

Nagin D, Tremblay RE. Trajectories of boys' physical aggression, opposition, and hyperactivity on the path to physically violent and nonviolent juvenile delinquency. Child Development. 1999; 70:1181-1196. [PubMed: 10546339]

Raine, A. The Psychopathology of Crime: Criminal Behavior as a Clinical Disorder. Academic Press; San Diego: 1993.

Raine A, Brennan P, Mednick SA. Birth complications combined with early maternal rejection at age 1 year predispose to violent crime at age 18 years. Archives of General Psychiatry. 1994; 51:984988. [PubMed: 7979887]

Rhee SH, Waldman ID. Genetic and environmental influences on antisocial behavior: a meta-analysis of twin and adoption studies. Psychological Bulletin. 2002; 128:490-529. [PubMed: 12002699]

Rosanoff AJ, Handy LM, Plesset IR. Criminality and delinquency in twins. Journal of Criminal Law and Criminology. 1934; 24:923-934.

SAS Institute. SAS OnlineDoc® 9.2. SAS Institute, Inc; Cary, NC: 2007.

Scher, KJ. Children of Alcoholics: A Critical Appraisal of Theory and Research. University of Chicago Press; Chicago: 1991. 
Sigvardsson S, Bohman M, Cloninger CR. Structure and stability of childhood personality: prediction of later social adjustment. Journal of Child Psychology and Psychiatry. 1987; 2:929-946. [PubMed: 3436998]

Swedish National Council for Crime Prevention. Victims' Tendency to Report Crime, Report. Vol. 2008. Brottsförebyggande rådet; Stockholm: 2008. p. 12

Tibbetts SG, Piquero AR. The influence of gender, low birth weight, and disadvantaged environment in predicting early onset of offending: a test of Moffitt's interactional hypothesis. Criminology. 1999; 37:843-878. 


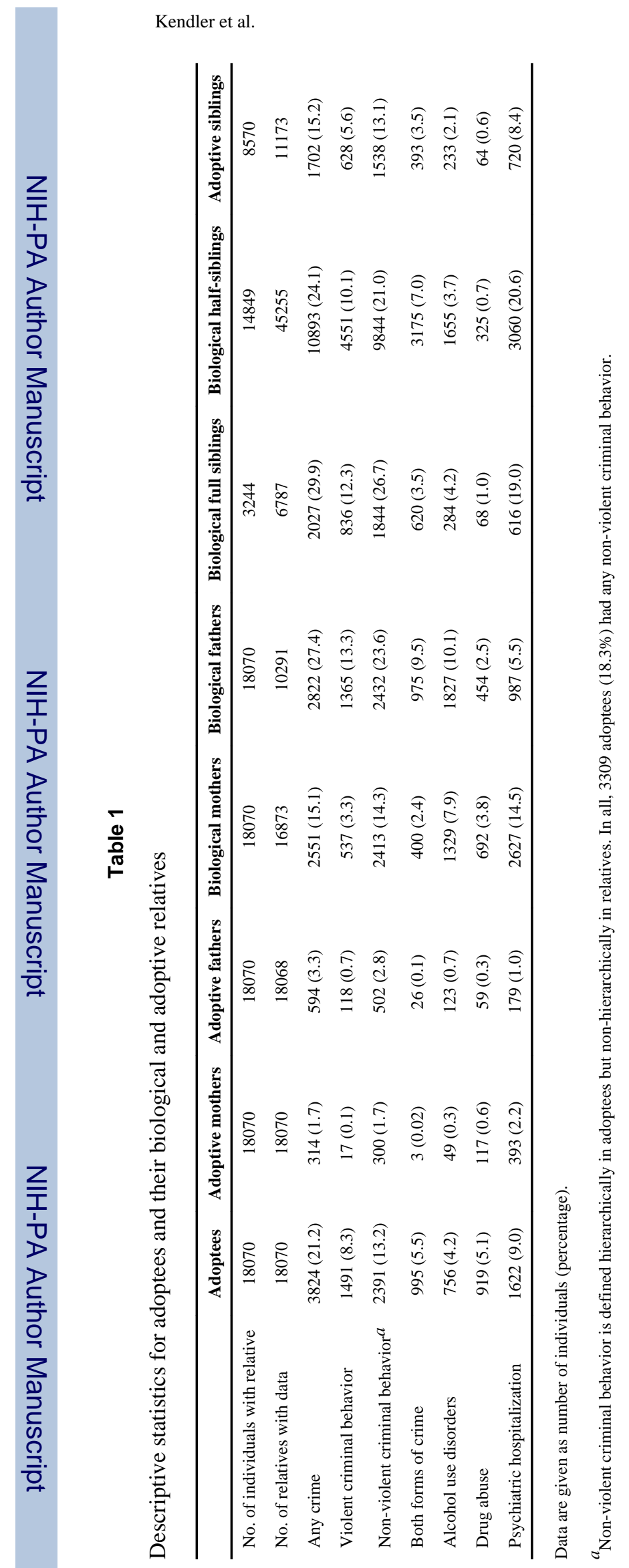

Psychol Med. Author manuscript; available in PMC 2014 October 01. 


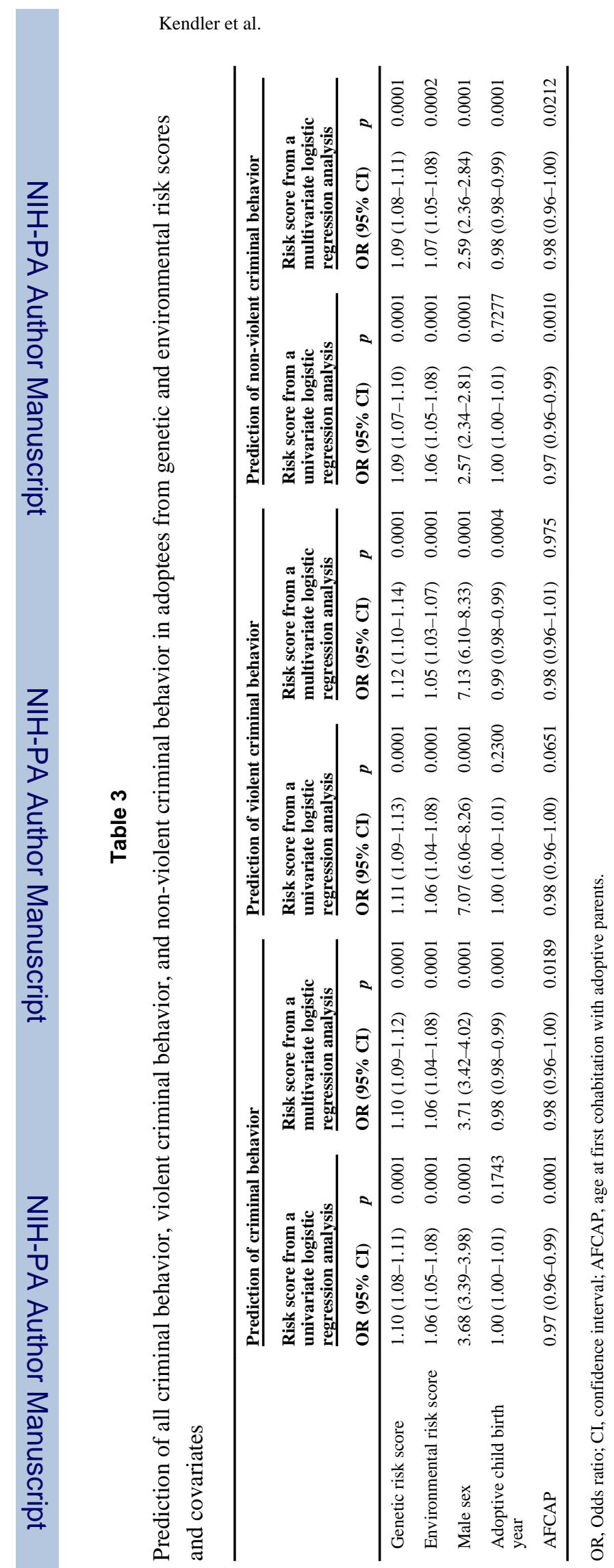

Psychol Med. Author manuscript; available in PMC 2014 October 01. 


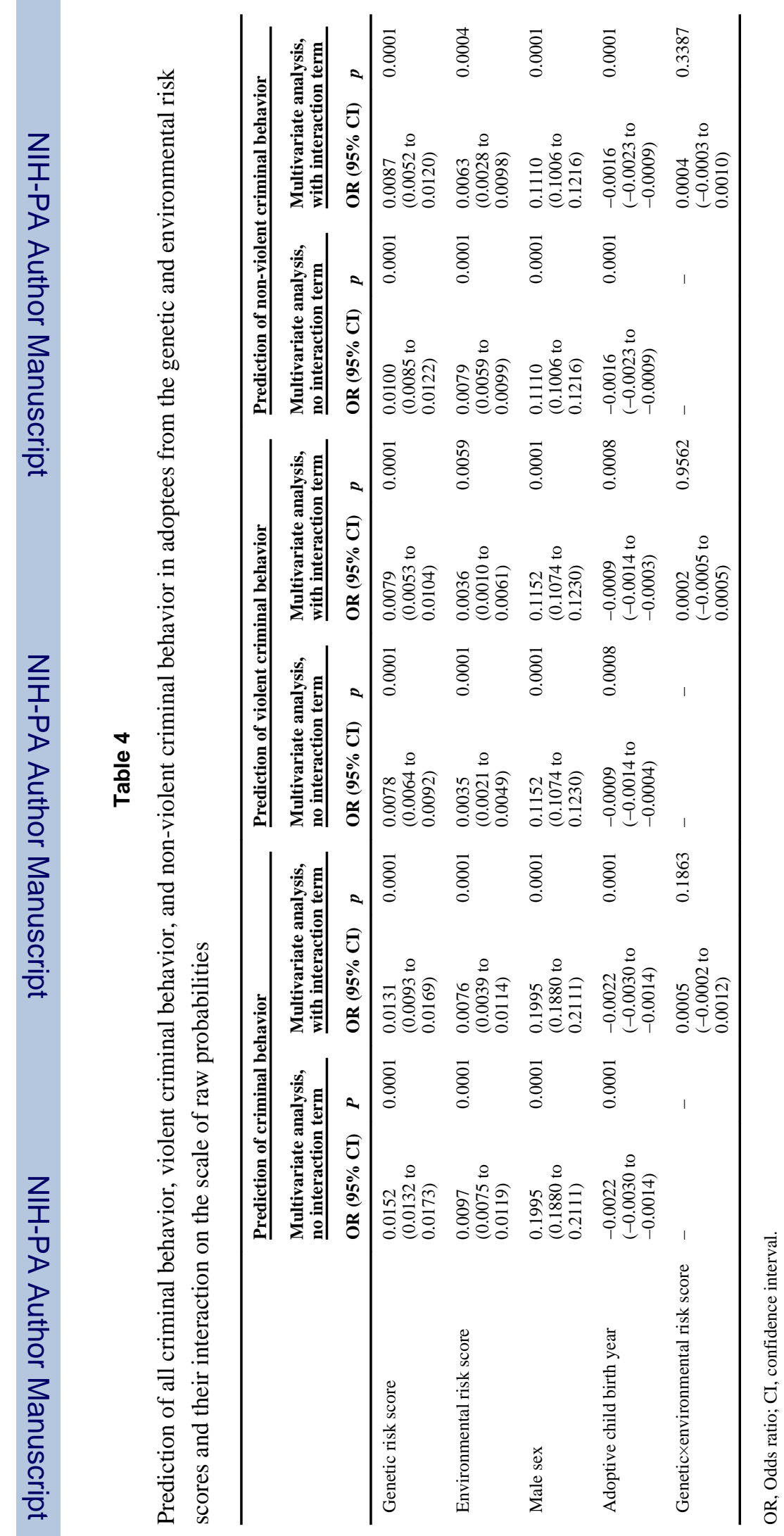


